TRANSACTIONS OF THE

AMERICAN MATHEMATICAL SOCIETY

Volume 232, 1977

\title{
DECOMPOSITION SPACES HAVING ARBITRARILY SMALL NEIGHBORHOODS WITH 2-SPHERE BOUNDARIES $\left({ }^{1}\right)$
}

\author{
EDYTHE P. WOODRUFF
}

\begin{abstract}
Let $G$ be an u.s.c. decomposition of $S^{3}$. Let $H$ denote the set of nondegenerate elements and $P$ be the natural projection of $S^{3}$ onto $S^{3} / G$. Suppose that each point in the decomposition space has arbitrarily small neighborhoods with 2-sphere boundaries which miss $P(H)$. We prove in this paper that this condition implies that $S^{3} / G$ is homeomorphic to $S^{3}$. This answers a question asked by Armentrout [1, p. 15]. Actually, the hypothesis concerning neighborhoods with 2-sphere boundaries is necessary only for the points of $P(H)$.
\end{abstract}

1. Introduction. Our main results are concisely stated in $\$ 3$. The proof is based on a shrinkability theorem, which is stated in \$4. The method of proving shrinkability uses a technique developed by Eaton [3] and modified for our use in $\S 6$. The proof of the main theorem is $\$ 5$.

In $\$ 7$ the notion of a defining sequence and a generalization of it tie our results to work of Harrold [5], Price [12], and Armentrout [2]. \$2 is terminology.

The author wishes to thank John J. Walsh for many very helpful and stimulating discussions.

2. Preliminaries. All decompositions used are upper semicontinuous by a standard definition such as in Whyburn [14]. For a decomposition $G$ of $S^{3}$, the set of nondegenerate elements is denoted by $H$, and the natural projection of $S^{3}$ onto $S^{3} / G$ by $P$. A subset $A \subset S^{3}$ is called saturated if for $g \in G$, either $g \cap A=\varnothing$ or $g \subset A$.

For the distance between sets $A$ and $B$ we use $\rho(A, B)$; for the closure of a set $A$ we use $\mathrm{Cl} A$; for its set theoretic boundary, interior, and exterior we use $\operatorname{Bd} A$, Int $A$, and Ext $A$, respectively; and for its $\varepsilon$-neighborhood we use $N(A, \varepsilon)$. For a collection $H$, let $H^{*}=\{x \in g: g \in H\}$.

A crumpled cube is the closure of either component of the complement of a (possibly wild) 2-sphere in $S^{3}$.

Let $A$ be an annulus bounded by 2 -spheres $S_{1}$ and $S_{2}$. A homeomorphism $\xi$

Received by the editors September 23, 1975 and, in revised form, February 3, 1976.

AMS (MOS) subject classifications (1970). Primary 54B15, 57A10; Secondary 57A45.

${ }^{1}$ This work was supported in part by a Trenton State College Faculty and Institutional Research Award.

- American Mathematical Society 1977 
taking $S_{1}$ onto $S_{2}$ is called admissible if there exists a homotopy $H: S^{2} \times I \rightarrow$ $A$ such that $H\left(S^{2} \times 0\right)=S_{1} ; H\left(S^{2} \times 1\right)=S_{2}$; and for $x \in S^{2}$ if $H(x \times 0)$ $=p \in S_{1}$, then $H(x \times 1)=\xi(p) \in S_{2}$.

\section{Statement of results.}

THEOREM 1. Suppose that for any $p \in S^{3} / G$ and open set $U$ containing $p$ there is an open set $V$ such that $p \in V \subset U$ and $\mathrm{Bd} V$ is a 2-sphere missing $P(H)$. Then $S^{3} / G$ is homeomorphic to $S^{3}$.

This theorem is the affirmative answer to a query of Armentrout [1, p. 15]. It is actually a corollary of the following stronger theorem, in which the hypothesis is restricted to points in $P(H)$.

THEOREM 2. Suppose that for any $p \in P(H)$ and an open set $U$ containing $p$ there is an open set $V$ such that $p \in V \subset U$ and $\mathrm{Bd} V$ is a 2-sphere missing $P(H)$. Then $S^{3} / G$ is homeomorphic to $S^{3}$.

4. Shrinkability. The basic concepts in the definition and theorem below were originally given in [10] and [11] by McAuley. His theorem is correct if the standard definition of u.s.c. is used. Concerning this, see the theorem by Reed in her thesis [13]. The version we use is from her work and provable using methods in [10]. More easily accessible to the reader may be papers by Edwards and Glaser [4], and Marin and Visetti [9]. Each of these has a shrinkability definition and a theorem with full proof; however, neither result is sufficient for our needs.

Definition. Suppose that $G$ is a decomposition of $S^{3}$. We say that $H$ is shrinkable in $S^{3}$ if for each saturated open cover $\mathcal{Q}$ of $H^{*}$, homeomorphism $\phi$ of $S^{3}$ onto $S^{3}$, and $\eta>0$; there exists a saturated open cover $W$ of $H^{*}$ that refines $Q$ and a homeomorphism $f_{\eta}$ of $S^{3}$ onto $S^{3}$ such that (1) $f_{\eta}=\phi$ off थ*, (2) for each $g \in H$, Diam $f_{\eta}(g)<\eta$, and (3) for each $W \in$ W there exists $U \in \mathcal{Q}$ such that $\phi(W) \cup f_{\eta}(W) \subset \phi(U)$.

Shrinkability Theorem (McAuley, Reed). If $H$ is shrinkable in $S^{3}$, then $S^{3} / G$ is homeomorphic to $S^{3}$.

Note that this definition does not require that $थ$ cover all of $S^{3}$. (The definitions used by Edwards and Glaser and by Marin and Visetti do require a cover of $S^{3}$.) The hypothesis of Theorem 1 yields a cover of $S^{3}$; whereas, the Theorem 2 hypothesis yields a cover of only $H^{*}$.

5. Reduction to our main lemma. In this section we reduce Theorem 2 to Lemma 1 below. We show that the hypothesis of Theorem 2 and Lemma 1 are enough to show the existence of the shrink required in the McAuley-Reed shrinkability theorem. The proof of Lemma 1 will be given in $\S 6$. 
Lemma 1. Suppose $G$ is an u.s.c. decomposition of $S^{3}, \varepsilon$ is a positive number, and $X$ is a crumpled cube in $S^{3}$ with $\operatorname{Bd} X$ a (perhaps wild) 2-sphere which fails to intersect each nondegenerate element of $G$. Then there exists $a$ homeomorphism $h: S^{3} \rightarrow S^{3}$ such that

(1) $h \mid S^{3}-N(X, \varepsilon)=1$,

(2) if $g \in G$ and $g \subset X$, then Diam $h(g)<\varepsilon$, and

(3) if $g \in G$, then Diam $h(g)<\varepsilon+$ Diam $g$.

Proof of Theorem 2. Let a saturated open cover $थ$ of $H^{*}$, a homeomorphism $\phi$ of $S^{3}$ onto $S^{3}$, and $\eta>0$ be given. For $g \in H$ consider $U_{g} \in \mathcal{U}$ containing $g$. There is a saturated open set $O_{g}$ such that $g \subset O_{g}$ and $\mathrm{Cl} O_{g} \subset U_{g}$. The image $P\left(O_{g}\right)$ is open in $S^{3} / G$. Hence, by hypothesis there is an open set $V_{g}$ such that $P(g) \in V_{g} \subset P\left(O_{g}\right), \mathrm{Bd} V_{g}$ is a 2-sphere, and (Bd $\left.V_{g}\right) \cap P(H)$ is empty. Now $P^{-1}\left(V_{g}\right)$ is a saturated open set containing $g$, and $P^{-1}\left(\mathrm{Bd} V_{g}\right) \subset U_{g}$ is a 2 -sphere missing $H^{*}$.

Since $\phi$ is uniformly continuous on $S^{3}$, there is a distance $\eta^{\prime}$ such that for $p, q \in S^{3}$, if $\rho(p, q)<\eta^{\prime} / 2$, then $\rho(\phi(p), \phi(q))<\eta / 2$. Let $H_{L}=\{g \in H$ : Diam $\left.g>\eta^{\prime} / 2\right\}$. Since $H_{L}^{*}$ is compact, there is a finite cover of it by saturated open sets of the form $P^{-1}\left(V_{G}\right)$. Call this finite cover $\mathscr{Q}_{1}$. Let $B$ denote $\left\{B d U_{1}: U_{1} \in U_{1}\right\}^{*}$. This is a closed set that misses $H^{*}$.

Let $\mathscr{Q}_{2}$ be the set of components of $S^{3}-B$ that are contained in $\mho_{1}^{*}$. From $\mho_{2}$ take a finite subcover $\mathscr{U}_{3}$ of $H_{L}{ }^{*}$. Note that $\mathscr{U}_{3}$ is an open cover of $H_{L}^{*}$ by separated sets; call them $U_{3}^{1}, U_{3}^{2}, \ldots, U_{3}^{m}$.

We now find a refinement $\vartheta_{4}$ of $\mho_{3}$ by the same procedure that we used

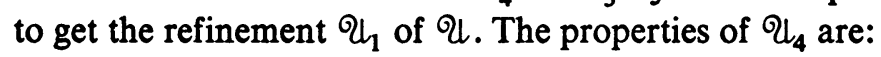

(a) it refines थ;

(b) it is a finite open cover of $H_{L}^{*}$;

(c) $\mho_{4}$ strictly refines $\mho_{3}$; i.e., for $U_{4} \in \mathscr{U}_{4}$, there is an element $U_{3} \in \mho_{3}$ which contains it and satisfies $U_{3} \supset \mathrm{Cl} U_{4}$;

(d) for $U_{4} \in \mathcal{U}_{4}, \mathrm{Bd} U_{4}$ is a 2-sphere which misses $H^{*}$; and

(e) although the elements of $U_{4}$ are not separated sets, given any $U_{3} \in U_{3}$, the union of those elements of $\mho_{4}$ lying in $U_{3}$ is separated from each other element of $\mho_{4}$.

Since the Shrinkability Theorem requires a cover $\mho$ of all of $H^{*}$, we will augment $\vartheta_{4}$ by the collection

$$
\mathcal{Q}_{4}{ }^{\prime}=\left\{\text { a component of }\left(\left(U \cap\left(S^{3}-\mathrm{Cl}{\mathcal{U}_{4}}^{*}\right)\right)-B\right): U \in \mathcal{U}\right\} \text {. }
$$

Let $\mho=\mathscr{Q}_{4} \cup \mathscr{\vartheta}_{4}{ }^{\prime}$. This saturated open cover $\mho$ of $H^{*}$ refines $\mathscr{Q}$ and also satisfies (e) above with $\mathcal{}$ replacing $\bigcup_{4}$. When (below) we perform homeomorphisms on crumpled cubes, we will move points outside those crumpled cubes. It is this condition (e) that will assure us that the shrinkability definition condition (3) will be satisfied. 
The shrinking homeomorphism $f_{\eta}$ will be the composition $\phi f^{m} \ldots f^{1}$, where each $f^{i}$ is a homeomorphism fixed off the corresponding $U_{3}^{i}$. The large nondegenerate elements in a set $U_{3}^{i}$ are covered by a collection $\left\{U_{4}^{1}, U_{4}^{2}, \ldots, U_{4}^{n}\right\} \subset \mathcal{Q}_{4}$. The homeomorphism $f^{i}$ will shrink the diameter of each nondegenerate element in $U_{3}^{i}$ to less than $\eta^{\prime}$. We will define $f^{i}$ as the composition $f_{n}^{i} \ldots f_{j}^{i} \ldots f_{1}^{i}$. Each $f_{j}^{i}$ will shrink diameters of elements in $U_{4}^{j}$ to a specified size and not allow diameters of other nondegenerate elements in $U_{3}^{i}$ to grow too much.

To define each $f_{j}^{i}$ we use Lemma 1. For $f_{1}^{i}$ the crumpled cube $X$ in $S^{3}$ is $\mathrm{Cl} U_{4}^{1}$. We choose $\varepsilon$ to be less than $\rho\left(U_{4}^{1}, \mathrm{Bd} U_{3}^{i}\right)$ and $\eta^{\prime} / 2$. Then $h$ gives us $f_{1}^{i}$. $\ldots$ For $f_{j}^{i}$ the crumpled cube is $\mathrm{Cl} f_{j-1}^{i} \ldots f_{2}^{i} f_{1}^{i}\left(U_{4}^{j}\right)$. We choose $\varepsilon$ to be less than $\rho\left(f_{j-1}^{i} \ldots f_{2}^{i} f_{1}^{i}\left(U_{4}^{j}\right)\right.$, Bd $\left.U_{3}^{i}\right)$ and $\eta^{\prime} /\left(2^{j}\right)$. Notice that use of $\eta^{\prime} /\left(2^{j}\right)$ controls the growth of the nondegenerate elements which have already been shrunk. The other condition on $\varepsilon$ makes $f_{j}^{i}$ be fixed off $U_{3}^{i}$. Now using $f^{i}=f_{n}^{i} \ldots f_{1}^{i}$ and $f_{\eta}=\phi f^{m} \ldots f^{1}$, we have a homeomorphism $f_{\eta}$ which satisfies the McAuley-Reed Shrinkability Theorem and proves Theorem 2 modulo Lemma 1.

6. Proof of Lemma 1. The proof of Lemma 1 is strongly influenced by Eaton's work [3]. Since a large portion of our proof is the observation that slightly more can be required of his shrink, the reader should be familiar with his paper before reading this section.

Consider $S^{3}$ to be the union of the crumpled cubes $X$ and

$$
K=\mathrm{Cl}\left(S^{3}-X\right) \text {. }
$$

Maps which we will define are indicated in the diagram.

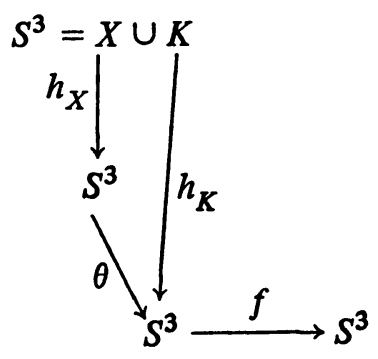

The map $H_{X}$ is a reembedding of $X$ in $S^{3}$. It is given by the Hosay-Lininger Theorem [6], [8], which states: If $C$ is a crumpled cube in $S^{3}$ and $e$ is a positive number, then there exists a homeomorphism $h$ from $C$ into $S^{3}$ such that $\mathrm{Cl}\left(S^{3}-h(C)\right)$ is a 3-cell and if $x \in C$ then $\rho(x, h(x))<e$. When we apply the theorem, we let $p \in \operatorname{Int} X$ and then require that $h_{X}$ is the identity on a small neighborhood of $p$. (The method of proof in [8] implies that this is possible.) 
Let $\rho(p, \operatorname{Bd} X)$ be denoted by $D$. Let $\theta$ be a homeomorphism of $S^{3}$ onto itself taking $h_{X}(X)$ to a set of diameter less than the minimum of $D / 2$ and $\varepsilon / 2$ and not moving points in a small neighborhood of $p$. Note that now all nondegenerate elements that were in $X$ are in $\theta h_{X}(X)$ and are small, and that $\theta h_{X}(X) \subset \operatorname{Int} X$.

We next apply the Hosay-Lininger Theorem to $K$ to get the reembedding $h_{K}$ of $K$ in $S^{3}$. Choose $e$ smaller than the minimum of $\varepsilon / 2$ and $\rho\left(K, \theta h_{X}(X)\right)$. We also require that $h_{K}$ be the identity on $S^{3}-N(X, \varepsilon)$. The $\varepsilon / 2$ condition controls growth of nondegenerate elements in $K$. The other condition on $e$ guarantees that $\theta h_{X}(X)$ and $h_{K}(K)$ do not intersect. Note that $\theta h_{X}(X)$ and $h_{K}(K)$ are disjoint crumpled cubes in $S^{3}$ and that the closure of the complement of each is a 3-cell. Denote $\mathrm{Cl}\left(S^{3}-\theta h_{X}(X)-h_{K}(K)\right)$ by $A$.

We will complete the proof of Lemma 1 by applying Lemma 2 , which we state here and will prove below.

Lemma 2. Suppose that in $S^{3}$ an annulus $A$ is bounded by 2-spheres $S$ and $\xi(S)$, where $\xi$ is an admissible homeomorphism on $S ; F_{1}$ and $F_{2}$ are disjoint 0 -dimensional $F_{\sigma}$-sets in $S$ such that $F_{1} \cup \xi\left(F_{2}\right) \cup \operatorname{Ext} A$ is 1-ULC; $U$ is an open set containing $A$; and $\varepsilon>0$. Furthermore, suppose that there is a decomposition $G$ of $S^{3}$ such that the nondegenerate elements miss $A$. Then there exists a map $f$ of $S^{3}$ onto $S^{3}$ such that

(1) $f \mid S^{3}-U=1$,

(2) $f \mid S^{3}-A$ is a homeomorphism onto $S^{3}-f(A)$,

(3) $f \mid S=f \xi$ and $f \xi$ is a homeomorphism onto $f(A)$, and

(4) for $g \in H$, Diam $f(g)<\varepsilon+\operatorname{Diam} g$.

We now show that the hypotheses of Lemma 2 are satisfied. The 2-sphere $S$ in Lemma 2 is $\theta h_{X}(\mathrm{Bd} X)$. The homeomorphism $\xi$ is $h_{K} h_{X}^{-1} \theta^{-1}$. Hence, $\xi(S)$ is $h_{K}(\mathrm{Bd} X)=h_{K}(\mathrm{Bd} K)$. For $U$ we use $\theta h_{X}(X) \cup A \cup N\left(h_{K}(\operatorname{Bd} K), \gamma\right)$, where $\gamma$ is such that for $p, q \in K$, we have $\rho\left(h_{K}(p), h_{K}(q)\right)<\gamma$ implies that $\rho(p, q)<\varepsilon$. We must show that $A$ is an annulus, that $h_{K} h_{X}^{-1} \theta^{-1}$ is admissible, and that the $F_{\sigma}$-sets exist.

The boundary of $A$ is the two copies of $\mathrm{Bd} X$. They are disjoint 2-spheres. Let $T$ be a tame 2-sphere in $A$ separating these boundary components. Consider a homeomorphism $\lambda$ of $\mathrm{Cl}\left(S^{3}-\theta h_{X}(X)\right)$ onto a polyhedral 3-cell $P$. Since $T$ is tame in $\mathrm{Cl}\left(S^{3}-\theta h_{X}(X)\right)$, it is bicollared in it. Its image is bicollared in $P$ and, hence, tame there. Since $\lambda \theta h_{X}(\mathrm{Bd} X)$ is $\mathrm{Bd} P$, it is tame. Hence, $\lambda(T)$ and $\mathrm{Bd} P$ bound an annulus. The homeomorphism $\lambda^{-1}$ must take this annulus back to an annulus. Similarly, the set bounded by $T$ and $\theta h_{K}(\mathrm{Bd} K)$ is an annulus. Thus, $A$ is the union of two annuli whose intersection is a 2-sphere in the boundary of each. This implies [7, p. 167] that $A$ is an annulus. 
Admissibility is concerned with preserving orientation. To guarantee that $h_{K} h_{X}^{-1} \theta^{-1}$ is admissible we made each of $h_{X}, h_{X}$, and $\theta$ have a neighborhood on which it is fixed. The following argument shows that this gives admissibility. Let $\delta_{X}$ and $\delta_{K}$ be tame 3-cells on which the reembeddings $\theta h_{X}(X)$ and $h_{K}(K)$, respectively, are fixed. We can make $\delta_{K}$ small enough that $S^{3}-\delta_{K}$ is a neighborhood of $X$. In $S^{3}-\delta_{K}$ - Int $\delta_{X}$ there are homotopies of Bd $X$ to $\mathrm{Bd} \delta_{X}$ and of $\theta h_{X}(\mathrm{Bd} X)$ to $\mathrm{Bd} \delta_{X}$. Similarly, in $S^{3}-\operatorname{Int} \delta_{K}-\delta_{X}$ there are homotopies of $h_{K}(\mathrm{Bd} X)$ to $\mathrm{Bd} \delta_{K}$ and of $\mathrm{Bd} X$ to $\mathrm{Bd} \delta_{K}$. Hence, in $S^{3}-$ Int $\delta_{X}-$ Int $\delta_{K}$ there is a homotopy of $\theta h_{X}(\mathrm{Bd} X)$ to $h_{K}(\mathrm{Bd} X)$. Since each of $\theta h_{X}(\mathrm{Bd} X)$ and $h_{K}(\mathrm{Bd} X)$ is tame on the side containing $A$, the homotopy can be pushed into $A$.

Eaton's Mismatch Theorem [3] implies that, since $X$ and $K$ are crumpled cubes whose intersection is the boundary of each and union is $S^{3}$, there exist disjoint 0-dimensional $F_{\sigma}$-sets $F_{1}^{\prime}$ and $F_{2}^{\prime}$ in $\mathrm{Bd} X$ such that $F_{1}^{\prime} \cup$ Int $X$ and $F_{2}^{\prime} \cup$ Int $K$ are 1-ULC. Hence, $\theta h_{X}\left(F_{1}^{\prime}\right)$ and $h_{K}\left(F_{2}^{\prime}\right)$ are the necessary $F_{\sigma}$-sets.

We have satisfied the hypotheses of Lemma 2 . The conclusion states that a certain map $f$ exists. Now the map

$$
h(x)= \begin{cases}f h_{K} & \text { for } x \in K, \\ f \theta h_{X} & \text { for } x \in X\end{cases}
$$

is the homeomorphism required for the conclusion of Lemma 1.

Proof of LemMa 2. This is similar to Eaton's proof of his Theorem 4. Indeed, one replaces his repeated applications of his Lemma 2 by one application of our Lemma 4 (below) and then repeated applications of our Lemma 3 (below). For $\gamma$ in Lemma 4 use $\varepsilon / 2$ and for $\gamma$ in the $j$ th repeated use of Lemma 3 use $\varepsilon /\left(2^{j+1}\right)$.

The following lemma is similar to Eaton's Lemma 2, but has the added hypotheses and conclusion involving $\gamma$ and the decomposition.

Lemma 3. Suppose that $C$ is a 3-cell in $S^{3}, D$ is a disk in $\mathrm{Bd} C, h$ is a homeomorphism of $D$ onto $\mathrm{Cl}((\mathrm{Bd} C)-D)$ such that $h \mid \mathrm{Bd} D=1, F_{1}$ and $F_{2}$ are disjoint 0-dimensional $F_{\sigma}$-sets in Int $D$ such that $F_{1} \cup h\left(F_{2}\right) \cup \operatorname{Ext} C$ is 1-ULC, $U$ is an open set containing $C-\operatorname{Bd} C, \beta>0$, and $\gamma>0$. Furthermore, suppose that there is a decomposition $G$ of $S^{3}$ such that the nondegenerate elements miss $C$. Then there exist a cellular subdivision $\left\{D_{1}, \ldots, D_{n}\right\}$ of $D$ with mesh less than $\beta$ and a map $f$ of $S^{3}$ onto $S^{3}$ such that

(1) $f \mid S^{3}-U=1$,

(2) $f \mid S^{3}-C$ is a homeomorphism onto $S^{3}-f(C)$,

(3) both $f \mid D$ and $f \mid h(D)$ are homeomorphisms,

(4) $\left(\cup \operatorname{Bd} D_{i}\right) \cap\left(F_{1} \cup F_{2}\right)=\varnothing$,

(5) $f(D) \cap f h(D)=f\left(\cup \mathrm{Bd} D_{i}\right)$,

(6) $f\left|\cup \operatorname{Bd} D_{i}=f h\right| \cup \operatorname{Bd} D_{i}$, 
(7) $f\left(D_{i}\right) \cup f h\left(D_{i}\right)$ bounds a 3-cell in $f(C)$ of diameter less than $\beta$, and (8) for $g \in H, \operatorname{Diam} f(g)<\gamma+\operatorname{Diam} g$.

Proof. We will modify Eaton's proof of his Lemma 2 to get condition (8). We will retain all of his proof and make the additions listed below. Every time a homeomorphism is performed on $S^{3}$, the size of the nondegenerate elements must be controlled. This will be accomplished in two ways. (a) The homeomorphism will be the identity off of a neighborhood containing only small elements. (Such neighborhoods of $C$ are available because the nondegenerate elements miss $C$.) (b) Each homeomorphism will move points only a small distance. When necessary, several such small homeomorphisms will be used with a new neighborhood for each.

For the $\varepsilon$ in the proof we use the minimum of the hypothesized $\beta$ and $\gamma / 4$.

In Eaton's proof, the fourth paragraph is the first one we need to change. We require that $V_{1}$ also satisfy the condition that it contain no nondegenerate element with diameter greater than $\gamma / 2$; for each $i>1$ no nondegenerate element in $V_{i}$ may have a diameter under $\alpha_{i-1} \ldots \alpha_{1}$ greater than $\gamma / 2$.

In Eaton's I 5 note that $\alpha=\alpha_{n} \ldots \alpha_{1}$ is a homeomorphism which is the identity on any nondegenerate element with diameter greater than $\gamma / 2$, and which causes growth of less than $\gamma / 2$ in the diameters of those nondegenerate elements which it does move.

In 78 replace $U$ by an open subset of it that contains no nondegenerate element with diameter (under $\alpha$ from Step 1) greater than $\gamma / 2$.

ๆๆ11, 12 involve the same care about nondegenerate elements as $\$ 4$.

In $\Uparrow 13$ the neighborhood $W$ must contain no nondegenerate element whose diameter under previous homeomorphisms has become as large as $\gamma / 2$.

In $\uparrow 15$ the same subset of $U$ that was used in $\ 8$ is now used.

In $¥ 20$ as we repeat parts $\mathrm{A}, \mathrm{B}$, and $\mathrm{C}$ we require that the nondegenerate elements in the image from previous work be small.

These changes complete the modified proof.

Since Lemma 3 applies to 3-cells and we have an annulus in Lemma 2, we need the following lemma to divide the annulus into two 3-cells.

LEMMA 4. Suppose that $A$ is an annulus in $S^{3}, S$ is a 2-sphere in $\mathrm{Bd} A$, $\xi$ is an admissible homeomorphism of $S$ onto $(\operatorname{Bd} A)-S, F_{1}$ and $F_{2}$ are disjoint 0 -dimensional $F_{\sigma}$-sets in $S$ such that $F_{1} \cup \xi\left(F_{2}\right) \cup \operatorname{Ext} A$ is 1-ULC, $U$ is an open set containing $A$, and $\gamma>0$. Furthermore, suppose that there is a decomposition $G$ of $S^{3}$ such that the nondegenerate elements miss $A$. Then there exist a simple closed curve $J$ in $S$ and a map $f$ of $S^{3}$ onto $S^{3}$ such that

(1) $f \mid S^{3}-U=1$,

(2) $f \mid S^{3}-A$ is a homeomorphism onto $S^{3}-f(A)$, 
(3) both $f \mid S$ and $f \mid \xi(S)$ are homeomorphisms,

(4) $J \cap\left(F_{1} \cup F_{2}\right)=\varnothing$,

(5) $f(S) \cap f \xi(S)=J$,

(6) $f|J=f \xi| J$,

(7) $f(S) \cup f \xi(S)$ bounds two 3-cells in $f(A)$, and

(8) for $g \in H, \operatorname{Diam} f(g)<\gamma+\operatorname{Diam} g$.

Proof. The proof is similar to that of Lemma 3. Here we squeeze a simple closed curve in $S$ to $\xi(S)$. Note that since we have no condition on the size of the 3-cells in (7), we can omit some of the procedures in Step 1 and all of Step 2.

By $\sigma_{2}$ we denote the boundary of a geometric cube with sides two units, by $a$ the geometric center of the cube, and by $\sigma_{1}$ the boundary of the geometric cube with center at $a$ and one unit sides, which are parallel to those of $\sigma_{2}$. Let $A^{\prime}$ denote the annulus bounded by $\sigma_{1}$ and $\sigma_{2}$. Let $p$ be the projection map of $A^{\prime}$ onto $\sigma_{1}$ that moves points on a radius toward $a$. Let $\hat{g}$ be a homeomorphism of $A^{\prime}$ onto $A$ such that $\hat{g}^{-1}(S)=\sigma_{1}$ and $p \hat{g}^{-1} \xi \hat{g} \mid \sigma_{1}=1$. (The admissibility condition on $\xi$ implies that such a homeomorphism $\hat{g}$ can exist.) Let $J^{\prime} \subset \sigma_{1}$ be a simple closed curve which is the boundary of a square separating $\sigma_{1}$ into two congruent parts. With $J^{\prime}$ used in place of Eaton's $G$, his argument shows that $\hat{g} p^{-1}\left(J^{\prime}\right)$ is tame. We let $J$ denote $\hat{g}\left(J^{\prime}\right)$. Corresponding to a distance $t \in[1,2]$ we let $L(t)$ denote the boundary of a geometric cube with side $t$ units and placed with respect to $\sigma_{1}$ as $\sigma_{2}$ is placed with respect to $\sigma_{1}$. Let $L(s, t)$ denote the closed annulus bounded by $L(s)$ and $L(t)$.

We complete the proof by pushing the tame graph $\xi \hat{g}\left(J^{\prime}\right)=\xi(J)$ along $\hat{g} p^{-1}\left(J^{\prime}\right)$ to the graph $\hat{g}\left(J^{\prime}\right)=J$. Care must be taken to be sure that this squeezing of $A$ does not cause nondegenerate elements to grow too much. The required map is the composition of a finite collection $\left\{\alpha_{i}\right\}$ of maps of $S^{3}$ onto $S^{3}$, obtained as follows. Let $\Delta t$ be a distance such that for any point $j \in J$, if $p^{-1}(j)$ contains two points $y$ and $z$ such that $\rho(y, z)<\Delta t$, then $\rho(\hat{g}(y), \hat{g}(z))<\gamma / 2$. Let $i=1, \ldots, n$, where $n$ is such that $(n-1) \Delta t<1<$ $n \Delta t$. Let $1=t_{0}, t_{i}=t_{i-1}+\Delta t$ if $0<i<n$, and $t_{n}=2$. Let $\alpha_{i}$ denote the projection map of $T_{i}=\hat{g}\left(p^{-1}(J) \cap L\left(t_{i-1}, t_{i}\right)\right)$ onto $J_{i}=\hat{g}\left(p^{-1}(J) \cap L\left(t_{i}\right)\right)$ defined by the relation $\alpha_{i}(x)=\hat{g}\left(p^{-1}\left(p \hat{g}^{-1}(x)\right) \cap L\left(t_{i}\right)\right)$. We extend the maps $\alpha_{i}$ to $S^{3}$ one at a time, as follows. Select a small regular neighborhood $V_{1} \subset U$ of $T_{1}$ that contains no nondegenerate element with diameter greater than $\gamma / 2$. Extend the map $\alpha_{1}$ to $S^{3}$ so that $\alpha_{1} \mid S^{3}-V_{1}=1$, so that $\alpha_{1} \mid S^{3}-$ $T_{1}$ is a homeomorphism onto $S^{3}-J$, and so that $\rho\left(\alpha_{1}, 1\right)<\gamma / 2$. Let $V_{2} \subset U$ be a small regular neighborhood of $T_{2}$ that contains no nondegenerate element whose image under $\alpha_{1}$ has diameter greater than $\gamma / 2$. Now extend $\alpha_{2}$ to $S^{3}$ so that $\alpha_{2} \mid S^{3}-V_{2}=1$, so that $\alpha_{2} \mid S^{3}-T_{2}$ is a 
homeomorphism onto $S^{3}-J_{2}$, and so that $\rho\left(\alpha_{2}, 1\right)<\gamma / 2$. Continuing thus, we define $V_{3}, \alpha_{3}, \ldots, \alpha_{n}$.

The map squeezing $A$ to two 3 -cells is the composition $f=\alpha_{n} \ldots \alpha_{1}$.

7. Discussion. A way of looking at the results of this paper is by generalizing the notion of a defining sequence. Recall that a sequence of manifolds with boundary $\left\{M_{i}\right\}$ in $S^{n}$ is called a defining sequence if $M_{i+1} \subset$ Int $M_{i}$ and the nondegenerate elements of the decomposition are the components of $\cap_{i=1}^{\infty} M_{i}$; a decomposition is definable by 3-cells if there is a defining sequence $\left\{M_{i}\right\}$ such that each $M_{i}$ is the union of disjoint 3-cells. Harrold [5] proved a theorem that is equivalent to the statement that $S^{3} / G$ is homeomorphic to $S^{3}$ if $G$ is definable by 3 -cells.

We make the following generalization of the notion of a defining sequence.

Definition. A decomposition $G$ of $S^{n}$ is locally definable by a manifold with boundary $M$ if for each nondegenerate element $g$ of $G$ there is a sequence $\left\{M_{i}\right\}$ of copies of $M$ such that (1) $M_{i+1} \subset$ Int $M_{i}$, (2) $\cap_{i=1}^{\infty} M_{i}=g$, and (3) Bd $M_{i}$ does not intersect any nondegenerate element.

Notice that for a decomposition $G$ satisfying this definition. $P(H)$ is 0-dimensional, but that $\mathrm{Cl} P(H)$ can be $S^{n}$.

Using this terminology, we can restate Price's theorem [12] as follows. If a decomposition $G$ of $S^{n}$ is locally definable by $n$-cells, then $S^{n} / G$ is homeomorphic to $S^{n}$.

In this paper we have proved that if a decomposition $G$ of $S^{3}$ is locally definable by crumpled cubes, then $S^{3} / G$ is homeomorphic to $S^{3}$.

Armentrout [2] has shown that for a sequence of crumpled cubes, conditions (1) and (2) of the locally definable definition are not sufficient to imply that $S^{3} / G$ is homeomorphic to $S^{3}$. This leads us to ask the following:

Question. Can condition (3) for a decomposition $G$ to be locally definable by crumpled cubes be weakened and it still be true that $S^{3} / G$ is homeomorphic to $S^{3}$ ?

\section{REFERENCES}

1. Steve Armentrout, Monotone decompositions of $E^{3}$, Topology Seminar (Wisconsin, 1965), Ann. of Math. Studies, no. 60, Princeton Univ. Press, Princeton, N.J., 1966, pp. 1-25. MR 36 \#5915.

2. A three-dimensional spheroidal space which is not a sphere, Fund. Math. 68 (1970), 183-186. MR 42 \#5239.

3. W. T. Eaton, The sum of solid spheres, Michigan Math. J. 19 (1972), 193-207. MR 46 \#8227.

4. Robert D. Edwards and Leslie C. Glaser, A method for shrinking decompositions of certain manifolds, Trans. Amer. Math. Soc. 165 (1972), 45-56. MR 45 \#4423.

5. O. G. Harrold, Jr., A sufficient condition that a monotone image of the three-sphere be a topological three-sphere, Proc. Amer. Math. Soc. 9 (1958), 846-850. MR 21 \#2223.

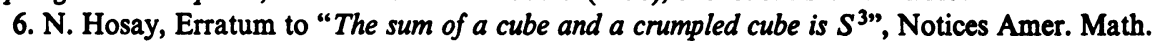
Soc. 11 (1964), p. 152. 
7. L. V. Keldyš, Topological imbeddings in Euclidean space, Proc. Steklov Inst. Math. 81 (1966); English transl., Amer. Math. Soc., Providence, R. I., 1968. MR 34 \# 6745; 38 \#696.

8. L. L. Lininger, Some results on crumpled cubes, Trans. Amer. Math. Soc. 118 (1965), 534-549. MR 31 \#2717.

9. A. Marin and Y. M. Visetti, A general proof of Bing's shrinkability criterion, Proc. Amer. Math. Soc. 53 (1975), 501-507.

10. Louis F. McAuley, Some upper semi-continuous decompositions of $E^{3}$ into $E^{3}, A n n$. of Math. (2) 73 (1961), 437-457. MR 23 \#A3554.

11. $\longrightarrow$ Upper semicontinuous decompositions of $E^{3}$ into $E^{3}$ and generalizations to metric spaces, Topology of 3-Manifolds and Related Topics (Proc. Univ. of Georgia Inst., 1961), Prentice-Hall, Englewood Cliffs, N. J., 1962, pp. 21-26. MR 25\#4502.

12. T. M. Price, $A$ necessary condition that a cellular upper semicontinuous decomposition of $E^{n}$ yield $E^{n}$, Trans. Amer. Math Soc. 122 (1966), 427-435. MR 33 \# 1843.

13. Myra J. Reed, Decomposition spaces and separation properties, Doctoral Dissertation, SUNY, Binghamton, 1971.

14. Gordon Thomas Whyburn, Analytic topology, Amer. Math. Soc. Colloq. Publ., vol. 28, Amer. Math. Soc., Providence, R.I., 1942. MR 4, 86.

Department of Mathematical Sciences, Trenton State College, Trenton, New Jersey 08625 